\title{
Identification of interstitial lung diseases using deep learning
}

\author{
Nidhin Raju, Anita H. B., Peter Augustine
}

Department of Computer Science, CHRIST (Deemed to be University), India

\begin{tabular}{l} 
Article Info \\
\hline Article history: \\
Received Dec 17, 2019 \\
Revised May 28, 2020 \\
Accepted Jun 6, 2020 \\
\hline
\end{tabular}

\section{Keywords:}

Classification

Deep learning

Medical image analysis

Neural network

\begin{abstract}
The advanced medical imaging provides various advantages to both the patients and the healthcare providers. Medical Imaging truly helps the doctor to determine the inconveniences in a human body and empowers them to make better choices. Deep learning has an important role in the medical field especially for medical image analysis today. It is an advanced technique in the machine learning concept which can be used to get efficient output than using any other previous techniques. In the anticipated work deep learning is used to find the presence of interstitial lung diseases (ILD) by analyzing high-resolution computed tomography (HRCT) images and identifying the ILD category. The efficiency of the diagnosis of ILD through clinical history is less than $20 \%$. Currently, an open chest biopsy is the best way of confirming the presence of ILD. HRCT images can be used effectively to avoid open chest biopsy and improve accuracy. In this proposed work multi-label classification is done for 17 different categories of ILD. The average accuracy of $95 \%$ is obtained by extracting features with the help of a convolutional neural network (CNN) architecture called SmallerVGGNet.
\end{abstract}

Copyright $\odot 2020$ Institute of Advanced Engineering and Science. All rights reserved.

\section{Corresponding Author:}

Nidhin Raju,

Department of Computer Science,

CHRIST (Deemed to be University),

Bengaluru, Karnataka, 560029-India.

Email: nidhin.raju@res.christuniversity.in

\section{INTRODUCTION}

Deep Learning is an excellent tool for feature learning, classifying, identifying, and quantifying patterns in medical images. The progressions of advanced graphics processing units (GPUs) and central processing units (CPUs), a large volume of dataset availability, and the development of many deep learning algorithms are some factors to the success of deep learning in every field. The deep learning techniques can effectively use when there is a massive amount of sample data are available in the training stage itself. The sufficient implementation of Deep Learning is in the extensive data set only. This is the main challenging task for applying deep learning in medical images to build a deep learning model with a minimal amount of training data [1]. The Medical Imaging methodology is effortless, non-obtrusive, and the vast majority of them do not require any extraordinary arrangement. Generally, to provide input to the deep learning models, two types of concepts are using; in the first case, the vector type values will be taken for multi-layer neural networks, and in another case, 2D or 3D image values will be taken for the convolutional networks. In this proposed work, a CNN architecture called smallerVGGNet, which is a subline of VGGNet used to classify the ILD category from 17 different categories.

Usually, ILD has incremental breathlessness, lung crackling, and an irregular chest x-ray. A variety of other illnesses, such as bacterial pneumonia, pulmonary edema, and malignancy (e.g., carcinomatosis lymphangitis), are identified as differential diagnoses [2]. Typically, tests for pulmonary function show lower volumes of lungs, poor gas transmission, and hypoxemia. Reducing the transfer factor and transport coefficient for carbon monoxide are typical of lung parenchyma diseases and their blood supply [3, 4]. 
Therefore, in ILD, but also emphysema and pulmonary vascular disease, these parameters are decreased. In emphysema, the chest x-rays usually show hyperinflated lungs, while, with reticulonodular infiltration, the ILD shows generally decreased lung volumes. Once ILD has suspected the first step is a thorough examination of the health history with a particular focus on occupational history (e.g., asbestos, coal dust), environmental exposure (e.g., bird touch, smoke from cigarettes), a list of all medications (e.g., amiodarone, methotrexate) and any symptoms that could include infectious diseases of the lungs [5]. The full medical history of a patient and any risk factors for an immunocompromised condition is essential as the clinical context depends on how the subsequent examinations are interpreted. In some instances, it is likely to have eosinophilia, autoantic, or avian precipitin.

It is cumbersome to diagnose the presence of ILD in a patient by clinical data and to go through all similar types of HRCT images of a patient since ILD encompasses many different pathological processes. The efficiency of the diagnosis of ILD through clinical history is less than $20 \%$. Currently, an open chest biopsy is the best way of confirming the presence of ILD. In the diagnostics of some ILDs, for example, Lung biopsy is a crucial component and is rarely required for the diagnosis of interstitial idiopathic pneumonia. A flexible bronchoscope can be performed simultaneously with bronchoalveolar lavage (BAL), and small sections of lungs are collected adjacent to the bronchi using transbronchial biopsies. A surgical biopsy requires general anesthesia with a complication rate of approximately $10 \%-20 \%$ and a mortality rate of less than $1 \%$ for the group of patients currently under selection. Many patients are deemed unfit for biopsy, and the possible benefits of assessing the histopathological history of the disease should be weighed against the procedural risks.

At this moment, the proposed method uses a deep learning architecture to categorize the ILD from HRCT images. The ILD comes with various categories, and almost every category looks like the same in HRCT images. It causes to clinicians to identify the exact parameters from those images. Sometimes it leads to confusion even for the doctors also to conclude. It will help in the inclusiveness of clinical evaluation for a better understanding of the disease. Once it detected, the treatment to the patients can get a start as soon as possible. If the clinical and HRCT features are typical of ILD, a biopsy may not be required. So, in this proposed work, we aim to categorize 17 categories of ILD from HRCT images by using a deep learning network named SmallerVGGNet.

The primary objective of this experiment is to find the presence of ILD by analyzing various HRCT images. HRCT gives greater accuracy than a chest radiograph for the diagnosis of ILD classification. Categories of ILD include nodules, thickened septa, reticulation, reduced attenuation areas, ground-glass opacities, honeycombing, and lymph nodes and pleura involvement in certain diseases. The presence of ILD in HRCT images can be ensured by analyzing its pattern because each category of ILD has different patterns in the HRCT image. Identification of the type of ILD is essential to treat the disease. There are many types of ILD. The proposed secondary objective is to categorize the ILD from 17 different types. The term ILD applies to a wide variety of more than 200 lung disorders. It is a crucial task for the clinicians to determine the parameters from HRCT images because even though ILD has a variety of patterns will look like the same for human eyes. The proposed deep learning technique will help to categorize the ILD from HRCT images.

\section{RELATED WORKS}

To the best of our knowledge, deep learning has not been reported to literature for the classification of ILD. In some approaches, there are used various deep learning concepts relevant to medical image analysis. The survey did mainly base on image classification, registration, segmentation, object detection, and other tasks. The most study was respected in the area of the breast, neuro, retinal, digital pathology, pulmonary, cardiac, abdominal, musculoskeletal [6]. From various studies, the authors found many things, such as the impact of deep learning algorithms in the analysis of medical images, challenges in analyzing, and benefits from this process. The best kind of models for the analysis of images to date were convolutional neural systems $(\mathrm{CNN})$. CNN's contained numerous layers that change their contribution with convolution channels of a little degree. In computer aid, deep convolutional systems had turned into the method of choice. The analysis of the medical image community network had paid heed to these crucial advancements.

The NiftyNet platform used to address the idiosyncrasies of medical imaging by supplementing the current deep learning infrastructure. Based on the TensorFlow library, the NiftyNet built. The TensorFlow library provided the tools for executing them efficiently on hardware resources and defining computational pipelines [7]. VGGNet emerged from the need to reduce and boost training time the number of parameters in the CONV layers. VGGNet (VGG16, VGG19, etc.) is available in multiple versions, which only differ in the total number of network components. The maximum parameter of VGG16 is 138 million. It is important to note here that all kernels in Conv are $3 \times 3$ size and 2x2 max pool with two stages. The purpose of a fixed kernel is to reproduce all variable-sized kernels used in Alexnet $(11 \times 11,5 \times 5,3 \times 3)$ using several $3 \times 3$ kernels as a building block. It improves on AlexNet by substituting large kernel filters (11 and 5 respectively in 
the first and second layer of convolution) with small kernel-sized $3 \mathrm{X} 3$ filters one after another. In this context, multiple smaller kernels, stacked, are better than a larger one, given the fact that multiple nonlinear layers increase the network depth that enables it to learn more complex features at a lower cost. The kernel has a different receptive field $[6,7]$.

For diagnosing and screening of many lung diseases, the chest X-ray is commonly using as the tool for the radiological examinations. The object segmentation and detection by deep learning produce better performance in the medical image analysis domain [8]. In medical imaging, the precise analysis, as well as evaluation of disease, relies upon both image interpretation and image acquisition. Image acquisition has improved considerably, finished late years, with gadgets gaining information at quicker rates and expanded goals. The image interpretation process, be that as it may, has as of late profited by computer technology [9]. polymyositis (PM) and dermatomyositis (DM) are foundational provocative disarranges with obscure etiology, furthermore, pathogenesis. They principally influence striated muscles, bringing about proximal muscle shortcoming. Polymyositis and dermatomyositis are extreme sickness elements influencing skeletal muscles and different organs, including the lungs. Interstitial lung disease (ILD) in PM/DM is progressively perceived as a genuine entanglement of the infection. ILD is a typical additional articular appearance of rheumatoid arthritis (RA), and a critical reason for bleakness and mortality in this patient populace [10, 11].

HRCT is broadly accessible, reliable in the hands of experienced radiologists, ease, and okay contrasted with a careful lung biopsy. Evaluation of the degree of radiological fibrosis loans extra prognostic esteem. The announced predominance of ILD in PM/DM in prior investigations generally fluctuates attributable to the absence of uniform symptomatic criteria for ILD, the different phases of the sickness in which patients were examined, and the wellspring of patient referral [12,13]. For a study, the authors could use analysis in 50 patients with biopsy-demonstrated NSIP, and a CT check was surveyed by two thoracic radiologists in accord. After the observations were portrayed, the eyewitnesses decided whether the observations were good with recently distributed portrayals of nonspecific interstitial pneumonia (NSIP) or whether the discoveries would bolster the conclusion of another unending infiltrative lung sickness. The CT observations in patients with NSIP and to contrast these and the CT discoveries of other perpetual infiltrative lung sicknesses were described [14].

Deep neural systems have, as of late, increased significant business enthusiasm because of the improvement of new variations of $\mathrm{CNNs}$ and the coming of proficient parallel solvers upgraded for present-day GPUs [15]. Notwithstanding, contrasted with 2D images for the most part utilized in computer vision, symptomatic and interventional images data in the medicinal field are frequently volumetric. This makes a need for calculations performing divisions in 3D by taking the entire volume content into the record without a moment's delay [16]. Preparing a deep CNN from scratch is troublesome because it requires a lot of labeled training data and a lot of aptitudes to guarantee appropriate intermingling. A promising option is to adjust a $\mathrm{CNN}$ that has been pre-trained utilizing, for example, an expansive arrangement of named standard images. Nonetheless, the significant contrasts among regular and medicinal images may exhort against such learning exchange [17].

The perfect biopsy procedure to distinguish men with prostate cancer would be findings of just significant prostate cancer growth and minimization of inconsequential prostate cancer detection and subsequent overtreatment. MRI-TBx is a promising system that may offer a portion of these focal points contrasted with standard efficient TRUS-Bx, as appeared direct examination of the two biopsy approaches in the analysis $[18,19]$. The analysis of prostate cancer differs from that in other strong organ cancer where imaging is utilized to distinguish those patients who require a biopsy. The prostate malignant growth demonstrative pathway offers transrectal ultrasound-guided biopsy (TRUS-biopsy) in men who present with a raised serum prostate-specific antigen (PSA) [20]. Imaging biomarkers (IBs) are essential to the standard administration of patients with the disease. IBS utilized day by day in oncology to incorporate clinical TNM arrange, target reaction, and left ventricular discharge division. Other CT, MRI, PET, and ultrasonography biomarkers are utilized widely in malignant growth research and medication improvement [21, 22]. New IBs should be built up either as helpful apparatuses for testing research speculations in clinical preliminaries and research examines or as clinical necessary leadership instruments for use in human services, by intersection 'translational gaps' through approval and capability [23, 24]. Endeavors to set up a quantitative way to deal with the CT-based portrayal of the lung parenchyma in interstitial lung malady (counting emphysema) has been looked at continuously. The exactness of these apparatuses must be site free. Multi-indicator push CT has remained the highest quality level for imaging the lung, and it gives the capacity to picture both lung structure just as lung work [25].

\section{IMPLEMENTATION}

\subsection{Data collection}

The data set comprises 3045 images of HRCT with three-dimensional annotated pathological lung tissue regions together with medical criteria of ILD conditions that have been confirmed pathologically. 
It contains 128 patients with one of thirteen ILD histological conditions, 108 image series of over 41 liters of annotated pulmonary tissue patterns, and a comprehensive collection of 99 medical parameters. It provides DICOM images and a .txt file with annotations. Then there is used Fileviewer Plus software to convert .roi to .jpg format as shown in Table 1. Later, we built an algorithm in Matlab to change the name of image files (because the same names were existing for many files). Each image contains $96 \mathrm{dpi}$ and 512 pixels for both width and height. The data organized in different classes according to the variety of ILD such as fibrosis, healthy, emphysema, ground glass, consolidation, micronodules, bronchial wall thickening, reticulation, cysts, macro nodules, peripheral micronodules, bronchiectasis, air trapping, increased attenuation, early fibrosis, tuberculosis, and PCP. It contained the common ILD categories were healthy, emphysema, groundglass, fibrosis, and micronodules as shown in Table 2.

Table 1. Dataset labels and the total number of images for each label with an example

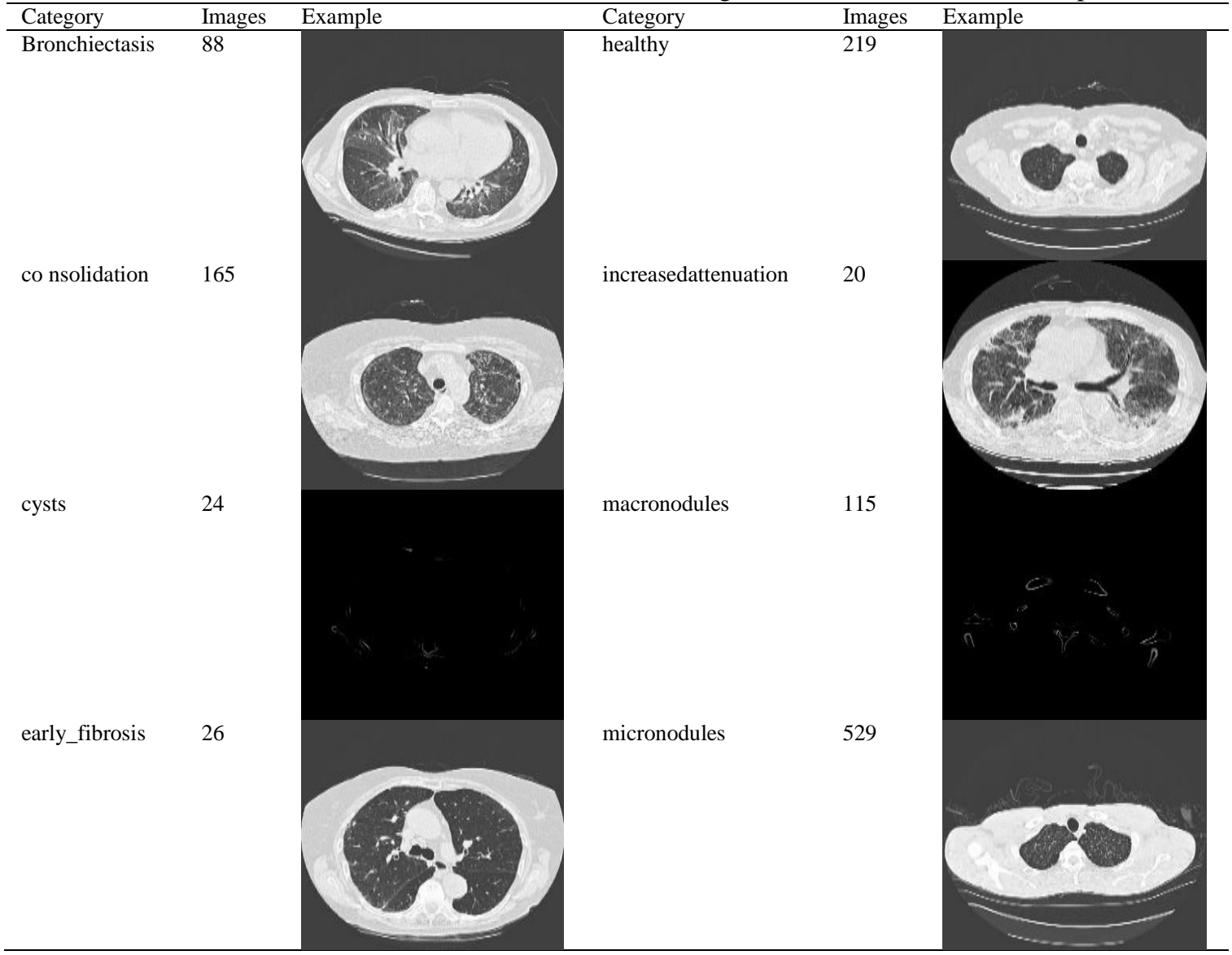

Table 2. Common ILD category and its description

\begin{tabular}{ll}
\hline Common ILD Category & Description \\
\hline healthy & Normal condition \\
emphysema & Emphysema is commonly used for rheumatoid arthritis-associated disorders related to \\
& tobacco smoking in patients with idiopathic pulmonary fibrosis (IPF) and ILD. \\
ground-glass & It is descriptive of an area in which CT with bronchial and vascular markings are more \\
& attenuated in the lung. \\
fibrosis & When damaged or scarred lung tissue \\
micronodules & Includes miliary tuberculosis and endobronchial disease dissemination \\
\hline
\end{tabular}

\subsection{Methodology}

SmallerVGGNet is the CNN architecture used for the proposed work, a streamlined version of its enormous sibling, VGGNet. The stages of the proposed work pictured in the given block diagram as shown in Figure 1. The entire implementation process has three stages. 


\subsubsection{Build the dataset}

The dataset creation algorithm built by including the Bing Image Search API of Microsoft, which was part of Microsoft's cognitive services, to bring AI to the text, speech, vision, and more for apps and software to build the thoroughly learned image datasets. After this period, if it needs to use for further implementation, then it required to pay some amount to google for the usage.

\subsubsection{Train the model}

The data augmentation is done by using a class called ImageDataGenerator, a method used to capture current images in our dataset and to produce extra training data using random transformations (rotations, shear, etc.). The augmentation of data used to avoid overfitting. Incremental improvements through backpropagation would contribute to the training of our Network for 75 EPOCHS. We set an initial learning rate as 3 (Adam Optimizer default value). We used a batch size of 32 for this project. The images will subsequently be resized as 96x96 with three channels. First, the algorithm loads each image into memory from disk. Then, it performs preprocessing by resizing images with width and height as 96 and then convert it into an array form. A multi-label classification task performed to split the path into several labels. A 2-element list is generated after this execution and then added to the labels. Then, convert the list to an array of NumPy and scale the pixel intensities to the range of [0,1]. Regarding the binarization of multi-class classification labels, the MultiLabelBinarizer class of the science-learn library used. Then these labels matched and transformed into a vector, which encodes the class(s) of the image. In machine learning practices, the next step is to divide the data for training and testing. $80 \%$ of the images are allocated for training, and $20 \%$ for testing. This then initialized the data augmentation object because the dataset contained less than 1,000 images for some categories. And next, the SmallerVGGNet model comes into the picture for the Multi-label classification. There used binary cross-entropy rather than categorical cross-entropy to compile the model. After the training completed, we saved the model and label binarizer to disk by training with our data augmentation generators. The accuracy and loss of the plot were stored as an image file from there.

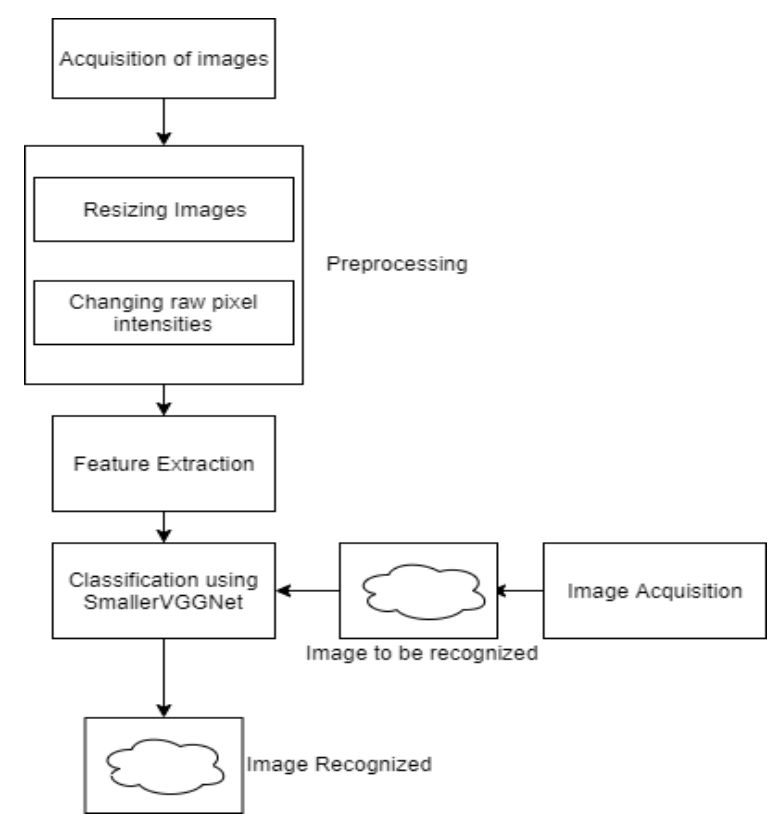

Figure 1. Block diagram of the proposed project

\subsubsection{Test the model}

Once CNN has been trained, it constructed a script that could identify objects that were not in training or validation/test collection. The pre-process of an input image is done in the same way as mentioned in the training phase. The model and multi-label binarizer are loaded from disk to memory by an algorithm. It classified the image and extracted the topmost labels based on indices. The array index is then sorted in descending order by its related probability, recording the topmost label indices, which therefore is the best one in the network. 
Algorithm-1

Input: HRCT images in .jpg format.

Output: Training loss and accuracy

1. Set the paths of dataset, model, MLB object and image plotting path

2. Load the images from dataset

3. Pre-process each images

a. resize the image into $96 * 96$

b. Change the raw pixel intensities of each image into the range $[0,1]$

4. Updating data list by extracting class labels (ILD category) and append it

5. Binarize labels with the advanced multi-label of scikit learn

6. Divide data into sets of training and testing using $80 \%$ of training data and $20 \%$ of test data.

7. Binary cross-entropy compile the model and then store the model and MLB to the disk

8. Store an image in disk with training loss and accuracy

\section{Algorithm-2}

Input: HRCT image in .jpg format.

Output: Predict ILD category with index

1. Set the paths of dataset, model, MLB object and image plotting path

2. Load the images from dataset

3. Pre-process each images

a. resize into $96 * 96$

b. Change the raw pixel intensities of each image into the range $[0,1]$

4. Load the CNN and MLB

5. Predict the category of input image with index of the category

6. Show the output by displaying input image with predicted category and corresponding index

\subsection{Experimental result}

In this experiment, the total number of 3045 HRCT images were processed. Each image was converted from .roi to .jpg format for the smooth execution of the system. Then, these images manually arranged in 17 different categories based on ILD categories for training the network. Once it has wholly trained, got accuracy, and validated the accuracy, 95\%, and 94\%, respectively as shown in Figure 2. Some sample images were applied to the trained network, and it could predict the categories successively, and its category prediction results are given as shown in Figures 3 and 4.

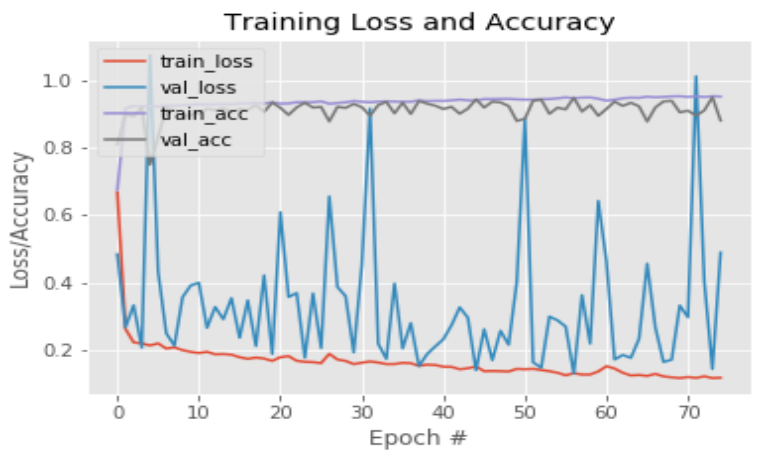

Figure 2. Graphical representation of training loss and accuracy

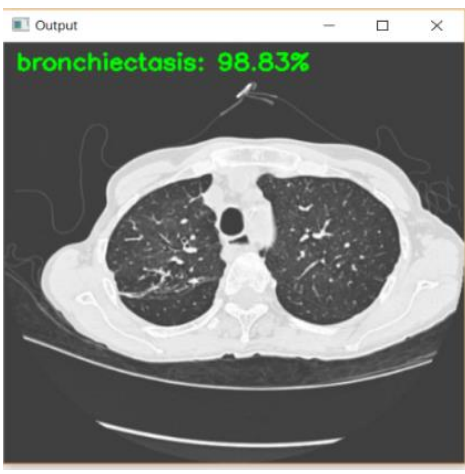

Figure 3. Predicted as bronchiectasis for a new input

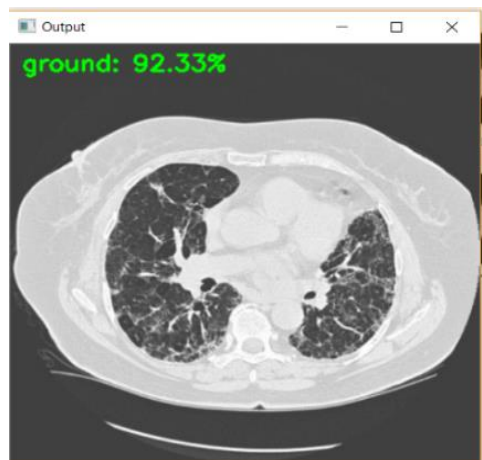

Figure 4. Predicted as fround for a new input 
The availability of the data for this experiment was minimal. The total number of 3045 HRCT images only could collect for this experiment. As it was a deep learning model, it requires a massive amount of data to train the model. The sufficient number of images per each class can produce a good result when it tested. The analysis of this work was done by creating 12 different models by considering the number of classes has taken and tested it with different images. In the first model, there have taken 17 classes and its graphically pictorised in Figure 5.

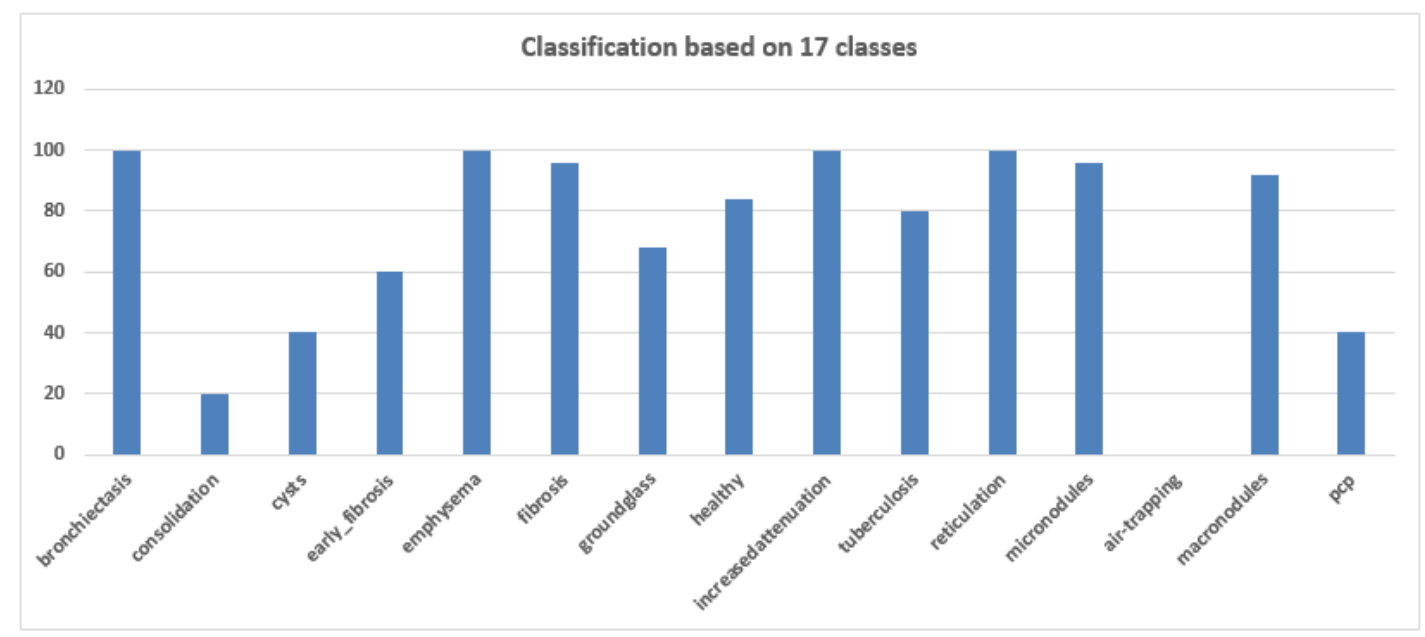

Figure 5. Graphical representation for classification of 17 classes

The next model built with five classes, which are the top common categories among 17 classes. In the remaining models, only two classes were considered, and for each model, picked up with two various classes from 5 popular classes as shown in Figure 6. When sample images tested with each model, there was showing accuracy variations in each model. The aim of creating models with two classes was that once if could able to find the top 2 classes from the 17 classes model, it can apply in 2 classes model, which was created by that top predicted classes. This activity can be used to clarify the disease once more.

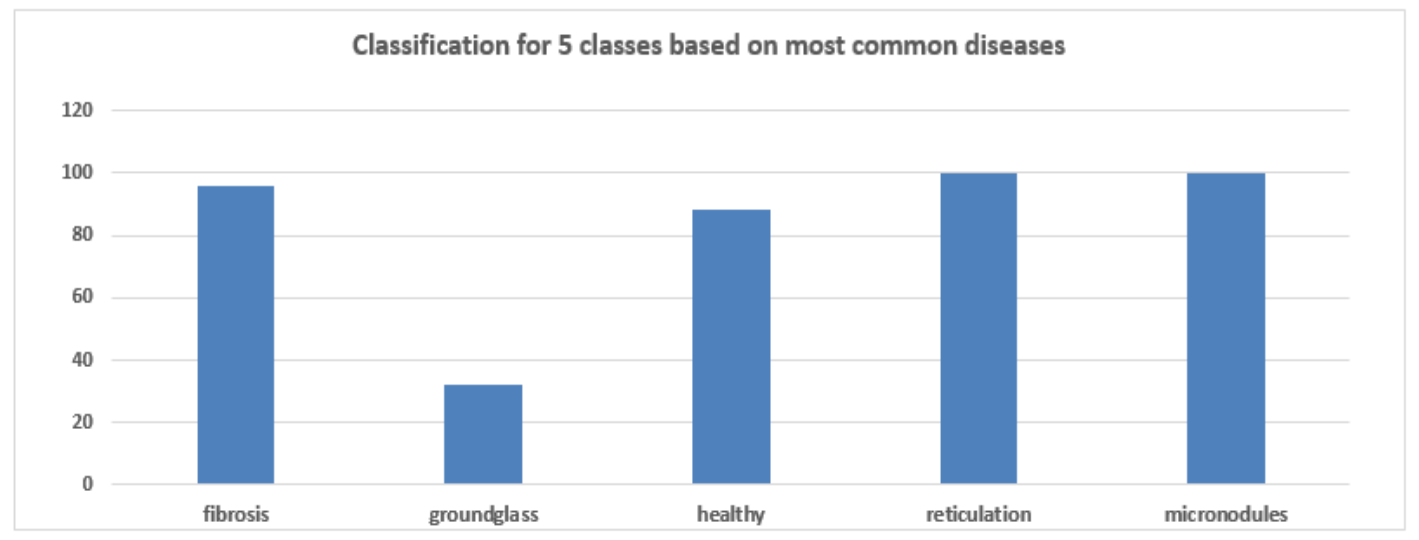

Figure 6. Graphical representation for classification of 5 classes

\section{LIMITATIONS}

The limited number of training data was one of the critical drawbacks of this algorithm. Ideally, when training a convolutional neural network, it required at least 500-1000 images per each class. Otherwise, it affected the accuracy of the result. One other disadvantage is that the system takes 2-3 hours to train the network. 


\section{CONCLUSION}

In this proposed work, a deep learning CNN architecture named SmallerVGGNet used to classify the ILD category from 17 different categories by processing HRCT images. For this experiment, 12 various deep learning models constructed according to the number of classes used for each model. The network with 17 classes could able to train the system with a 95\% accuracy rate. After that, a few numbers of input images were submitted to the trained the system, and it could classify the ILD categories successively. The remaining models also created based on the diseases which have been occurring most commonly. Once the topmost disease category can be able to identify, again, it can check with the sub-models for better clarity. After applying some samples on the procedure mentioned above, it showed some variation in predicting the result. Based on the availability of data for each class, the models generated different results. The models in which classes contained a right amount of data gave a good result in predicting the ILD category than models in which classes included less amount of data. Hence this study can be processed for early-stage detection of ILD for better treatment to the patients. In the future, this system can be utilized to execute the system without resizing the images and to apply more filters to increase the accuracy of the result.

\section{REFERENCES}

[1] Işın, Ali, Cem Direkoğlu, and Melike Şah, "Review of MRI-based brain tumor image segmentation using deep learning methods," Procedia Computer Science, vol. 102, pp. 317-324, 2016.

[2] Liu, Siqi, et al. "Early diagnosis of Alzheimer's disease with deep learning," IEEE 11th international symposium on biomedical imaging (ISBI), pp. 1015-1018, 2014.

[3] Liskowski, Pawel, and K. Krawiec, "Segmenting retinal blood vessels with deep neural networks," IEEE Transactions on Medical Imaging, vol. 35, no. 11, pp. 2369-2380, 2016.

[4] Scholl, Ingrid, Aach, T., Deserno, T. M., and Kuhlen, T., "Challenges of medical image processing," Computer science-Research and development, vol. 26, no. 1-2, pp. 5-13, 2011.

[5] Weese, Jürgen, and Cristian Lorenz, "Four challenges in medical image analysis from an industrial perspective," Elsevier, no. 44-49, 2016.

[6] Litjens, Geert, et al., "A survey on deep learning in medical image analysis," Medical image analysis, vol. 42, pp. 60-88, 2017.

[7] Gibson, Eli, et al., "NiftyNet: a deep-learning platform for medical imaging," Computer methods and programs in biomedicine, vol. 158, pp. 113-122, 2016.

[8] Wang, Xiaosong, et al. "Chestx-ray8: Hospital-scale chest x-ray database and benchmarks on weakly-supervised classification and localization of common thorax diseases," Proceedings of the IEEE Conference on Computer Vision and Pattern Recognition, pp. 2097-2106, 2017.

[9] Greenspan, Hayit, Bram Van Ginneken, and Ronald M. Summers, "Guest editorial deep learning in medical imaging: Overview and future promise of an exciting new technique," IEEE Transactions on Medical Imaging, vol. 35 , no. 5 , pp. $1153-1159,2016$.

[10] Spampinato, Concetto, et al., "Deep learning for automated skeletal bone age assessment in X-ray images," Medical image analysis, vol. 36, pp. 41-51, 2017.

[11] Kim, Eunice J., et al. "Usual interstitial pneumonia in rheumatoid arthritis-associated interstitial lung disease," European Respiratory Journal, vol. 35, no. 6, pp. 1322-1328, 2016.

[12] Fathi, Maryam, et al., "Interstitial lung disease, a common manifestation of newly diagnosed polymyositis and dermatomyositis," Annals of the rheumatic diseases, vol. 63, no. 3, pp. 297-301, 2004.

[13] Lee, Yoon Jin, et al., "Hepatocellular carcinoma: diagnostic performance of multidetector CT and MR imaginga systematic review and meta-analysis," Radiology, vol. 275, no. 1, pp. 97-109, 2016.

[14] Hartman, Thomas E., et al., "Nonspecific interstitial pneumonia: variable appearance at high-resolution chest CT," Radiology, vol. 217, no. 3, pp. 701-705, 2000.

[15] Kang, Eunhee, Junhong Min, and Jong Chul Ye, "A deep convolutional neural network using directional wavelets for low-dose X-ray CT reconstruction," Medical physics, vol. 44, no. 10, 2017.

[16] Milletari, Fausto, Nassir Navab, and Seyed-Ahmad Ahmadi, "V-net: Fully convolutional neural networks for volumetric medical image segmentation," Fourth International Conference on 3D Vision (3DV), pp. 565-571, 2016.

[17] Tajbakhsh, Nima, et al. "Convolutional neural networks for medical image analysis: Full training or fine tuning?," IEEE transactions on medical imaging, vol. 35, no. 5, pp. 1299-1312, 2019.

[18] Avendi, M. R., Arash Kheradvar, and Hamid Jafarkhani, "A combined deep-learning and deformable-model approach to fully automatic segmentation of the left ventricle in cardiac MRI," Medical image analysis, vol. 30, pp. 108-119, 2016.

[19] Schoots, Ivo G., et al., "Magnetic resonance imaging-targeted biopsy may enhance the diagnostic accuracy of significant prostate cancer detection compared to standard transrectal ultrasound-guided biopsy: A systematic review and meta-analysis," European urology, vol. 68, no. 3, pp. 438-450, 2015.

[20] Ahmed, Hashim U., et al., "Diagnostic accuracy of multi-parametric MRI and TRUS biopsy in prostate cancer (PROMIS): A paired validating confirmatory study," The Lancet, vol. 389, pp. 815-822, 2017. 
[21] Smith-Bindman, Rebecca, et al., "Use of diagnostic imaging studies and associated radiation exposure for patients enrolled in large integrated health care systems, 1996-2010," Jama, vol. 307, no. 22, pp. 2400-2409, 2012.

[22] Yin, Xiao-Xia, Sillas Hadjiloucas, and Yanchun Zhang, "Introduction and Motivation for Conducting Medical Image Analysis," Pattern Classification of Medical Images: Computer Aided Diagnosis, pp. 1-26, 2017.

[23] Pham, Dzung L., Chenyang Xu, and Jerry L. Prince, "Current methods in medical image segmentation," Annual review of biomedical engineering, vol. 2, no. 1, pp. 315-337, 2000.

[24] O'connor, James PB, et al., "Imaging biomarker roadmap for cancer studies," Nature reviews Clinical oncology, vol. 14, no. 3, pp. 169-186, 2017.

[25] Hoffman, Eric A., et al., "Characterization of the interstitial lung diseases via density-based and texture-based analysis of computed tomography images of lung structure and function1," Academic radiology, vol. 10, no. 10, pp. 1104-1118, 2003.

\section{BIOGRAPHIES OF AUTHORS}
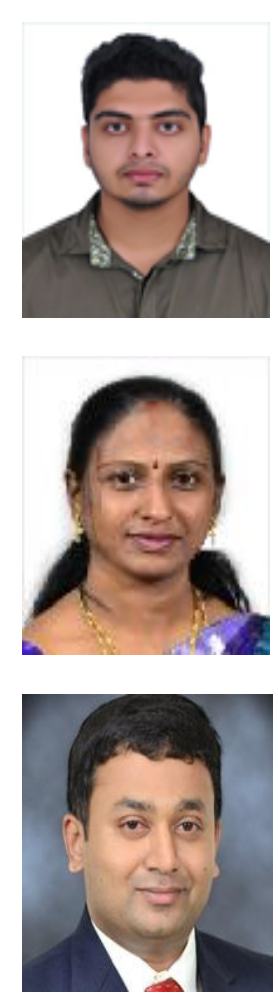

Nidhin Raju has been working as an Associate Software Engineer in Centre for Digital Innovation, CHRIST (Deemed to be University), India. He perusing M.Phil in Computer Science from CHRIST (Deemed to be University), India. His research interest is Image processing and Deep Learning. He has published a research paper in an International journal.

Email: nidhin.raju@ res.christuniversity.in

Dr. Anita H. B. is working as Associate Professor in the Department of Computer Science at CHRIST (Deemed to be University). She has received a PhD from Gulbarga University, India, in 2014. MCA degree from Indira Gandhi National Open University in 2003. Her research interest is Image processing, Sound processing and Machine Learning. She has published many papers in international and national conference and published two Book chapters in Springer Link, India. Also, she has published many research papers in International journals.

Email: anita.hb@christuniversity.in

Peter Augustine has been working as an Associate Professor in the Department of Computer Science, CHRIST (Deemed to be University), Bangalore. Peter Augustine has a PhD in Medical Image Processing in Cloud Environment, with over 8 years in cloud computing and 5 years in Big Data Analytics. He has authored various research papers published in peer-reviewed journals. He has been involved in a Major Research Project using Cloud Computing which costs more than 18 lakhs. He has also collaborated with St. John's Medical Research Institute for the research project to diagnose Lung Diseases using cutting-edge AI and Machine Learning. His research interests include artificial intelligence, IoT and big data analysis in the area of Healthcare and data mining, and human computer interaction. He has written chapters for the books focusing on some of the emerging technologies such as IoT, Data Analytics and Science, Blockchain and Digital Twin.

Email: peter.augustine@ christuniversity.in 